\title{
¿QUÉ ES ANTIFILOSOFÍA? UNA CONSIDERACIÓN SEGÚN LACAN, BADIOU Y LA FILOSOFÍA EDIFICANTE DE RORTY
}

\author{
WHAT IS ANTIPHILOSOPHY? A CONSIDERATION ACCORDING TO LACAN, \\ BADIOU AND THE EDIFYING PHILOSOPHY OF RORTY
}

\author{
Alejandro Cavallazzi Sánchez* \\ Universidad Iberoamericana, \\ Ciudad de México D.F. - México. \\ Recibido enero de 2019/Received January, 2019 \\ Aceptado marzo de 2019/Accepted March, 2019
}

\begin{abstract}
RESUMEN
Este trabajo muestra las definiciones postuladas por Lacan y Badiou respecto al término "antifilosofía" con el primer objetivo de mostrar una concepción integral del término y en segundo lugar compararlo con la "fillosofía edificante" presentada por Rorty. La antifilosofía es un discurso que no se opone al discurso filosófico sino que tiene un objetivo distinto. Si la filosofía se ocupa desinteresadamente del conocimiento, la antifilosofía tiene siempre presente el interés del individuo. Siguiendo la lectura psicoanalítica (Lacan), filosófica (Badiou, Rorty) y estética (Groys) pretendemos mostrar una unidad conceptual del término antifilosofía.
\end{abstract}

Palabras Clave: Antifilosofía, Lacan, Badiou, Rorty.

\begin{abstract}
This paper shows the definitions postulated by Lacan, Badiou and Groys regarding the term "antiphilosophy". The first objective is to present an integral conception of the term. The second objective is compare the notion of antiphilosophy with "edifying philosophy" as presented by Rorty. Antiphilosophy is a discourse that does not oppose philosophical discourse but has a different objective. If philosophy disinterestedly deals with knowledge, the antiphilosophy always keeps the interest of the individual in mind. Following the psychoanalytic (Lacan), philosophical (Badiou, Rorty) and aesthetic reading (Groys) of the term we intend to show a conceptual unity of the concept of antiphilosophy.
\end{abstract}

Key Words: Antiphilosophy, Lacan, Badiou, Rorty.

"Pero, ¿me dirás: qué pasa con la filosofía en todo esto? Bueno, la filosofía es lo que no sucede" (Badiou, 2013b, p 55). ${ }^{1}$

\section{EL ORIGEN DEL TÉRMINO EN JACQUES LACAN}

A mediados de los años setenta Jacques Lacan lanza una provocación: el término antifilosofía como marco de sus enseñanzas (Tomsic, 2016). Lacan lo utilizó en su día, en 1974, como una conjetura; era la época en que había un Departamento de

Psicoanálisis en París VIII, y entre las disciplinas posibles en la formación del analista -haciendo la salvedad de que no se trataba de especializar al psicoanalista en nada-, habló en aquel entonces de lingüística, topología, antifilosofía.

* Autor correspondiente / Corresponding author: alejocava@gmail.com 
Lacan propone a la antifilosofía como el área de estudio de aquellos que se han expuesto al psicoanálisis en el contexto de una educación universitaria (Johnston, 2010). El pasaje donde Lacan se refiere al término es bastante parco: "intitularía [la antifilosofía como] la investigación de lo que el discurso universitario debe a su posición 'educativa'. No es la historia de las ideas, tan triste ella, la que la llevará a cabo" (Lacan, 2012, p. 335). El término no tuvo posteriormente en Lacan una especial presencia (Alemán, 2001). No obstante, y a pesar de eso, esta aportación dio pie a una importante discusión a partir de su recuperación por Alain Badiou.

La elección del término resultaba entonces, como ahora, bastante contraintuitiva e históricamente problemática dado que fue utilizado en el siglo XVIII para referirse a los pensadores anti-ilustrados franceses (Masseau, 2010, como se citó en Tomsic, 2016, p. 81). Sin embargo, esta antifilosofía lacaniana no tiene la connotación negativa, despectiva y peyorativa contra la filosofía como la defendida por los reaccionarios de los tiempos de la revolución. Sería difícil pensar este antagonismo si consideramos la insistencia y el respeto con que Lacan recurre a la filosofía de manera positiva para explorar y expandir temas propios al punto que no sería exagerado considerarlo el psicoanalista más filosófico del siglo veinte (Sharpe, 2015, p. 2$)^{2}$.

El propio término 'antifilosofía' puede conducir de inmediato a equívocos como quien interpreta que "la posición antifilosófica es tal debido a que la antifilosofía está en contra de la filosofía, esto significa que la audiencia 'A' con el que el antifilósofo se enfrenta es filosófica" (Djordjevic, 2009, p. 27) ${ }^{3}$. Lacan no tiene el interés de criticar a la filosofía aunque, en ocasiones, se expresó con dureza a la epistemología de nuestro tiempo caracterizada, aún y según él, por un representacionalismo de corte kantiano.

Ya en 1972, en 'L 'étourdit' estigmatizó con el término 'imbecilidad' al kantismo persistente de todo 'lo que piensa' de nuestro tiempo. Con eso se refería a los filósofos u hombres de ciencia inducidos por la 'topología inepta' de Kant, que dice sólo conocer fenómenos. Esta imbecilidad es la negación de lo real. (Soler, 2006, pp. 123-124) ${ }^{4}$.
Lacan, más que una crítica a la filosofía (pues esto lo hubiera convertido ipso facto en filósofo), buscaba mostrar una carencia de la perspectiva filosófica y sobre todo resaltar un contraste entre su propio método psicoanalítico y la filosofía.

La antifilosofía no es antifilosófica en el sentido de ser 'no-filosófica'; por el contrario, el antifilósofo no pretende evadir o destruir la filosofía, sino llamar la atención sobre formas de conocimiento que la filosofía no puede conocer, al enfrentar la filosofía y subvertir sus afirmaciones. (Clemens, 2013, p. 2) ${ }^{5}$.

A pesar del equívoco al que puede conducirnos el término antifilosofía la elección es etimológicamente afortunada ya que el prefijo 'anti-' más allá de contrariedad tenía el significado de sustitución.

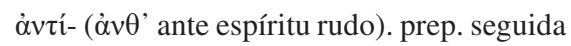
por el gen. Significado original, opuesto. De allí otros significados son: delante o enfrente de, contra, para, en lugar de, a cambio de, al igual de, etc. Para indicar que una persona o cosa toma o ha de tomar el lugar de otra: en lugar de, a cambio de, etc.: Mt. 2:22; Lc. 11:11; Jn. 1:16; He. 12:2; He 12:16; Stg. 4:15. (Tuggy, 1996, § 473).

Por ejemplo el 'anti-cristos' más allá de ser alguien que se pone a Cristo es aquel que ocupa su lugar. De forma análoga, y tal como es planteado por Lacan, la antifilosofía vendría a ocupar, en ciertas ocasiones, el lugar de la filosofía más que oponerse.

El 'anti' que veo en el trabajo en el estudio antifilosófico (de Lacan)sobre la ansiedad debe entenderse en la misma línea que los cristianos ortodoxos tienden a leer el 'anti' en el Anticristo: lo que le interesa no es tanto el asalto directo, sostenido y totalmente visible a las doctrinas filosóficas establecidas sino el reemplazo sutil de esto último por algo nuevo e imperceptible, pero innegablemente diferente. (Robertson, 2015, pp. 12-13) ${ }^{6}$.

El seguimiento que hizo Badiou del término original de Lacan sigue esta concepción.

Badiou distingue antifilosofía de filosofía con base a su actitud, si no de su contenido 
o método. Es un tipo de pensamiento que se constituye, si hemos de creer a Badiou, en una 'hostilidad' a las pretensiones sistemáticas de la filosofía propiamente dicha. (Tanke, 2009 , p. 217) 7 .

Aquí debe resaltarse que esta hostilidad se dirige a las pretensiones de sistematizar de la filosofía y no hacía a la filosofía misma. La antifilosofía "implementa las herramientas lógicas y analíticas de la filosofía contra sí mismo en un esfuerzo por señalar sus suposiciones tácitas o fallas sistemáticas" (Tanke, 2009, p. 217) ${ }^{8}$ de tal suerte que la antifilosofía es caracteriza por "aquellas formas de pensamiento que postulan un acto fundador intuitivo o inefable, tal como se puede encontrar en la crítica de Kierkegaard a Hegel"".

Estos desarrollos se elaboraron a partir de lo poquísimo que Lacan se refirió al término. Pensadores posteriores como Badiou se dieron a la tarea de reconstruirlo teóricamente. Al respecto observamos al menos tres comprensiones. La primera es la homologación de la filosofía con el discurso del amo, la segunda el correlato filosófico entre el ser y el pensar y la tercera la diferencia que establece entre la verdad y el conocimiento.

Primero. Lacan piensa su antifilosofía en el contexto particular de su propia teoría. Existen en Lacan cuatro tipos de discurso: el discurso del amo, del histérico, del analista y el discurso de la universidad (Lacan, 1992). Lacan asocia este último discurso con el de la historia de la filosofía (Tomsic, 2016, p. 92; Johnston, 2010, p. 140) ${ }^{10}$ que genera un conocimiento que se hace pasar por una ideología disimulada que acomoda los intereses del amo en turno (Johnston, 2010, p. 140).

La filosofía sería una manera de buscar conocimiento que, a pesar del famoso argumento platónico donde el filósofo sale de la caverna política-histórica (Rep. VII) no obstante se mantendría sujeto de la necesidad, si no del deseo, del gobierno político. (Sharpe, 2015, p. 9) ${ }^{11}$.

Cabe aclarar que para Lacan la filosofía no es un sinónimo del discurso del amo sino que opera en sus linderos. La filosofía dice lo que el amo calla, opera como el bufón de la corte ante el rey.
El filósofo es, por lo tanto, quien gira en el discurso del maestro. Él juega el papel del loco. Es decir, el lugar de la verdad, absolutamente inconsciente de lo que dice, y que, en consecuencia, está limitado a jugar esta parte. (Badiou, 2013b, p. 22) ${ }^{12}$.

La antifilosofía se opondría, por supuesto, a esta manera de pensar de la filosofía aunque se trate de una filosofía asociada con una forma muy particular de discurso: el del discurso del amo. "La teoría de los discursos de Lacan contempla en la universidad un entrelazamiento del capitalismo y el conocimiento científico, y en su contexto sociopolítico el auge del poder burocrático y la proliferación de los expertos" (Tomsic 2016, p. $92)^{13}$. A este respecto Lacan identifica algo que los propios filósofos son conscientes, al menos, desde Marx aunque dicho por él en términos de economía política: la infraestructura determina la superestructura.

La asociación de la filosofía con el discurso del amo, por parte de Lacan, es reduccionista en cuanto a la pluralidad de las prácticas y argumentaciones filosóficas (Sharpe, 2015, p. 9) ${ }^{14} .14$ No obstante, se puede excusar a Lacan precisamente por que su interés no es calificar a toda la filosofía sino marcar una distancia respecto de su propio discurso. Al respecto Badiou comenta, el objeto de la antifilosofía no es antagonizar la filosofía sino situarse en una posición distinta: "los analistas deben leer la filosofía, pero ponerla a prueba bajo la ley fundamental del discurso analítico, y así leerla para no entrar en la filosofía, sino para escapar de ella" (Badiou, 2013b, p. 48) . $^{15}$.

La filosofía referida por Lacan, que no es toda filosofía, se asocia en el discurso de la universidad (supeditada al discurso del amo) mientras que el psicoanálisis se posiciona en el discurso del analista (Lacan, 2008) ${ }^{16}$.

Encontramos una segunda distinción en el correlato que la filosofía encuentra entre el ser y el pensar tal y como se encuentra a lo largo de toda su historia desde Parménides, pasando por Aristóteles, la definición escolástica de verdad Veritas est adcequatio intellectus et rei, continuando hasta la modernidad y nuestro tiempo. En cambio, la verdad en psicoanálisis se encuentra escindida o rota entre el sujeto y el mundo. "Para el psicoanálisis como antifilosofía 'hay una relación con el ser que no puede ser conocida'. Lacan, evocando 
un fragmento de Parménides dice: 'la discordancia entre conocimiento y ser es mi sujeto" (Sharpe, 2015 , p. 15$)^{17}$. Cabe decir que existen posturas filosóficas donde la relación del ser tampoco puede ser conocida como en el psicoanálisis; notablemente la filosofía kantiana. Sin embargo, la diferencia radica en que el psicoanálisis opera precisamente a partir de esta imposibilidad mientras que para la filosofía la imposibilidad es algo que permanece fuera del discurso, una terra ignota. Siguiendo la expresión coloquial de los programadores It's not a bug, it's a feature la falta de correlato entre ser y pensamiento no es una falla o algo por responder en psicoanálisis, como lo sería en el discurso filosófico, sino que es justamente su premisa.

Una tercera distinción entre filosofía y antifilosofía radicaría en la diferencia entre verdad y conocimiento. El psicoanálisis describe la oposición de la mayoría de los asuntos humanos entre la verdad y el engaño y no el paradigma filosófico que concierne a la verdad y la falsedad o el error. Esta oposición verdad-engaño es más bien marginal en el discurso filosófico (Sharpe, 2015). La verdad que interesa al sujeto de enunciación que se encuentra en falta (manque) no puede ser objetificada ni enunciada: mucho menos en un discurso filosófico o científico (Sharpe, 2015).

El discurso filosófico (...) excluye de la consideración teórica la dimensión específica de lo simbólico, central para el psicoanálisis. El lenguaje para los filósofos es el medio de la revelación, de ahí el posible adequatio o inadecuación (en error, ilusión y falsedad) con lo que es real. Para el psicoanálisis lacaniano, por el contrario, las palabras o significantes son en primer lugar los símbolos ofrecidos de una buena fe fundante entre sujetos, de ahí el intercambio simbólico en el que el engaño y la traición se hacen posibles y en el que se modela cada uno de nuestros deseos. (Sharpe, 2015, p. 22) ${ }^{18}$.

La antifilosofía junto con la lingüística, la matemática y la topología son las disciplinas de las cuales se vale el psicoanálisis para su quehacer particular (Lacan, 1992) y que conforman la lista de disciplinas que deben ser incluidas en la formación del analista que Samo Tomsic llama el quadrivium antiphilosophicum (Tomsic, 2016, p. 91). Para el psicoanalista Jorge Alemán: "la antifilosofía no es una especialidad, como no debe haber una especialidad en topología o lingüística, sino que es una de las diversas maneras de hablar de aquello en lo que consiste la experiencia analítica" (Alemán, 2001, p. 3). En este sentido, la antifilosofía sería un modo de hablar, una disciplina que se sirve de la filosofía para los propósitos del psicoanálisis.

\begin{abstract}
Se trata del límite entre el sentido, el campo del lenguaje -con sus efectos de significación y comprensión- y la pulsión, verdadero representante de lo real del cuerpo en el campo del psiquismo, una frontera que separa y une a la vez. Una bisagra entre palabra y goce. (Tutivén, 2002, p. 53).
\end{abstract}

La práctica del psicoanálisis se da precisamente en esta frontera. "La antifilosofía es un modo de hablar de la experiencia psicoanalítica y una discusión del modo de transmitirla" (Tutivén, 2002, p. 53). Para Lacan la antifilosofía es un modo de describir la práctica psicoanalítica que no se opone radicalmente a la filosofía, sino que se ubica en su lindero y se sirve de su discurso para su propio propósito.

Hemos mostrado cómo el término 'antifilosofía' sirve a Lacan para distanciar su postura de la filosófica. No obstante, desde su misma morfología la 'antifilosofía' se encuentra en relación con la filosofía: si bien negativa. Filosofía y psicoanálisis hablan exactamente de lo mismo, aunque "en términos tanto más idénticos cuanto que apuntan a un efecto opuesto" (Milner, 1995, p. 160). La relación filosofía-antifilosofía sirve a modo de contraste para mostrar un aspecto del psicoanálisis, pero el término antifilosofía qua antifilosofía resulta ajeno al interés del psicoanálisis. No se encuentra, ni tiene porqué encontrarse, en Lacan ni en los lacanianos una comprensión de la antifilosofía como un campo o estudio autónomo, sino que permanece como un modo de entender el psicoanálisis. No obstante, el atrevimiento lacaniano abre un nuevo campo de comprensión de lo filosófico que debe ser examinado al margen de los intereses de la clínica psicoanalítica.

\section{LA ANTIFILOSOFÍA EN ALAIN BADIOU}

La relación filosofía-antifilosofía permanece en el ámbito del psicoanálisis si nos quedamos con lo poco que Lacan llegó a decir al respecto y la reconstrucción teórica del término antifilosofía 
realizada por los lacanianos. No obstante, estas elaboraciones dieron lugar a que Alain Badiou adoptara el término y le diera un tratamiento filosófico ${ }^{19}$.

Badiou trabajó el término extensivamente a lo largo de tres seminarios: sobre la antifilosofía y Nietzsche (1992-93) (Badiou, 2015), antifilosofía $y$ Wittgenstein (1993-94) (Badiou, 2013a) para culminar con antifilosofía y Lacan (1994-95) (Badiou, 2013b). Finalmente, el tratamiento del tema dio lugar para la publicación original adicional de San Pablo. La fundación del universalismo (Badiou, 1999).

La comprensión de Badiou sobre la antifilosofía no está limitada a lo que sería la difícil sino imposible reconstrucción del término por Lacan. En cambio, la categoría emerge como el nombre de una larga tradición de pensadores que respecto a la tendencia filosófica dominante de su tiempo se sitúan en la extraño posición topológica de estar "afuera con" o de una "exterioridad interna". (Boostels, 2008, p. 158).

Badiou identifica a una serie de pensadores bajo el mote de antifilósofos entre los que destacan los pensadores de su seminario: Nietzsche, Wittgenstein y Lacan, pero también San Pablo, Pascal, Rousseau y Kierkegaard (Boostels, 2008).

Pero ¿qué característica tendrían estos pensadores en común para ser reunidos bajo la misma categoría de antifilosofía? No sólo un autor tendría que ser crítico respecto a la tendencia filosófica dominante de su tiempo, sino que tendría que cuestionar la suposición básica que se puede encontrar a lo largo de la historia de la filosofía donde

la cuestión del ser, o la del mundo, es coextensiva con la cuestión del lenguaje; en consecuencia, la reducción de la verdad a ser nada más que un efecto lingüístico o retórico; el resultado de específicos juegos de lenguaje históricos o culturales. (Boostels, 2008, p. 161) ${ }^{20}$.

Mientras que desde Parménides encontramos la reducción del ser al pensar, la antifilosofía se pregunta la posibilidad que no todo puede ser dicho y que hay algo, o más bien mucho, que escapa al lenguaje.
Badiou retoma y se apoya en la distinción lacaniana entre verdad (verité) (Johnston, 2010) y saber (savoir). A su vez, también conviene distinguir entre saber y conocer (connaîtrelconnaissance). El primero (saber/savoir) es una comprensión conceptual o intelectual, el segundo (conocer/ connaîtrelconnaissance) se trata de una conciencia que implica familiaridad y cercanía (Johnston, $2010)^{21}$. "Las verdades del inconsciente, situadas en el registro de lo Real, desafían el conocimiento (connaissance) pero no el saber (savoir) analítico" (Johnston, 2010, p. 149) ${ }^{22}$. El psicoanálisis debe valerse de la formulación intelectual del saber (savoir), a través del uso del matema (la fórmula que representa de manera simbólica los términos de la estructura), pues el conocimiento (connaissance) está inexorablemente atado al destino de otros dos términos la verdad y lo real.

Lacan integra la verdad y el conocer con un tercer elemento que corresponde a uno de sus célebres registros: lo real. Esto real se define como la falta de sentido ( $a b$-sense) por lo que la conjunción verdad/saber/real debe ser pensada en el ámbito del sentido (Badiou, 2017).

"En la reconstrucción de Lacan por Badiou de este período, el conocimiento de que no es conocimiento (conaissance) ni verdad (verité) (aunque mantiene una relación con este último) es lo que toca el ab-sense" (Johnston, 2010, pp. 149-150) ${ }^{23}$. Este $a b$-sense debe distinguirse del non-sense. El término $a b$-sense alude a la ausencia (absense) y lo que está ausente es precisamente el sentido (sense). En la esfera de lo real lacaniano, donde no puede haber imagen ni lenguaje, lo que está ausente es precisamente el sentido. "Lo real puede ser definido como sense qua ab-sense. Lo real es $a b$-sense, por tanto ausencia de sentido" (Badiou, 2017, p. 49 $)^{24}$. La ausencia de sentido (ab-sense) no implica lo que coloquialmente conocemos como el sin sentido (non-sense) que se refiere a lo absurdo. Cuando se observa un absurdo ( $a$ nonsense) se presenta en un campo de significaciones donde un elemento en particular está fuera de lugar ${ }^{25}$. El non-sense presupone un campo de significación mientras que $a b$-sense es la falta total de sentido (sense) y sin sentido (non-sense). En la ab-sense no cabe ni lo uno ni lo otro. Debe quedar claro de la distinción $a b$-sense/non-sense es que Lacan no apela a ningún existencialismo o irracionalidad. Lacan no es un absurdista (Badiou, 2017). Lo 
que se está señalando es que lo real es ausencia o substracción de sentido.

Una vez presentadas las anteriores distinciones se aprecia mejor la diferencia entre filosofía y antifilosofía.

Para Lacan la operación filosófica consiste en mostrar que hay un significado de la verdad. Como es objetiva, la consolación que nos ofrece bajo el término 'sabiduría' es ser capaz de decir que hay una verdad de lo real. Se da el axioma implícito o explícito: hay significado de la verdad porque hay verdad de lo real. (Badiou, 2017, p. 49) ${ }^{26}$.

En cambio la posición en psicoanálisis es que no hay significado de la verdad porque no hay verdad de lo real. Cuando se trata de lo real solamente hay un conocimiento de una función (Badiou, 2017, p. 49). "La relación de lo real que Lacan propone como la del discurso del analista será una relación del significado del conocimiento qua ab-sex sense, mientras que la relación filosófica de lo real es el del registro de la verdad" (Badiou, 2017, p. 51) ${ }^{27}$.

El registro de lo real lacaniano escapa del ámbito de la filosofía incluso cuando se trata de dar un significado de su conocimiento. La filosofía sería incapaz de asir el principio por el cual algo está relacionado con el conocimiento de la función de lo real (Badiou, 2017) ${ }^{28}$. Dicho de una forma más sencilla la filosofía está "atrapada en la pareja sentido-verdad que asume que el opuesto del sentido (sense) es el sin sentido (non-sense) y no la ausencia de sentido (ab-sense)" (Badiou, 2017 , p. 51 ${ }^{29}$. La filosofía es tanto incapaz como reacia a reconocer e incorporar la ausencia de sentido (ab-sense) (Johnston, 2010) ${ }^{30}$.

Lo anterior nos permite mostrar tres características de la filosofía que la distinguen de la antifilosofía:

Primero, la filosofía carece de consciencia alguna sobre el registro de la $a b$-sense. No quiere saber nada de este registro. La filosofía invariablemente substituye algo más por el $a b$-sense o el $a b$-sex sense.

Segundo, la filosofía no sabe nada de la función del conocimiento en lo real. Lo absorbe como amor a la verdad.

Y tercero, hay una cualidad especial para la filosofía porque ordena el significado y la verdad como imágenes en un espejo bajo el pretexto de decir que hay, posiblemente, un significado de la verdad. (Badiou, 2017, pp. 54-55) ${ }^{31}$.

En cambio, la $a b$-sense es una parte integral del psicoanálisis lacaniano y por extensión de la antifilosofía. Para Lacan no hay verdad de lo real (sólo hay verdad en tanto hay una función de lo real en el conocimiento), tampoco un conocimiento de lo real (no es lo mismo que la función de lo real en el conocimiento) ni conocimiento de la verdad. Lo que importa en cambio es que la tríada conocimiento-verdad-real no puede ser rota; no se puede acomodar en parejas. Cuando se habla de una pareja (como conocimiento de la verdad) siempre queda implicado y aludido el tercer término (en este caso lo real) (Badiou, 2017, p. 55).

La filosofía en cambio busca romper la triada en y acomodar los términos en parejas porque asume que puede haber un conocimiento de la verdad (correlato ser-pensar) pero esto ignora la categoría lacaniana de lo real. La filosofía no puede aceptar que la tríada conocimiento/verdad/ real es irreductible.

Lo real es imposible de conocer (connaître) precisamente porque está empotrado en la tríada verdad-conocimiento-real y no puede extraerse de ella para emparejarse con uno de los otros dos términos. Incluso se tiene que decir sobre lo real que "suplanta conocimiento' (dépose le connaître). Lacan llama a esta suplantación de conocer la 'demostración' de lo real, que es una palabra bastante extraña, aunque convincente. Lo real no puede ser conocido; debe ser demostrado. (Badiou, 2017, p. 58) $)^{32}$.

Esta demostración tiene un sentido particular. Significa que al no poder haber una ciencia de lo real, lo real debe ser figurado a través de una formalización lógica, es decir, a través del matema que son las fórmulas propias del lenguaje psicoanalítico lacaniano. Como lo de lo real no se puede decir o representar, nos debemos contentar con su formalización. No hay lenguaje de lo real sólo fórmulas (Badiou, 2017).

La segunda forma en que se demuestra lo real es el modo en el que se abre a la dimensión del acto. Como no hay conocimiento (connaisance) ni verdad de lo real y como la verdad solamente se presenta a través de una función del saber (savoir) 
sólo queda un encuentro puro con lo real. El punto en el que se da el encuentro con lo real es el acto (Badiou, 2017) ${ }^{33}$.

A la filosofía no podría importarle menos esta decisión externa, en la que el acto constituye la incisión silenciosa de una verdad desconocida, ya que el sello distintivo temporal de la filosofía es que tiene todo el tiempo del mundo. Esto siempre ha servido para identificar en su discurso la conexión antidialéctica que tiene con la eternidad de la Verdad. (Badiou, 2017, pp. 61-62) ${ }^{34}$.

En cambio, el psicoanálisis lacaniano no tiene ningún compromiso en mantener la conexión entre conocimiento y verdad porque la verdad está atada al registro de lo real y es en el dispositivo analítico bajo la forma del matema que se produce el acto que interesa al análisis ¿Cómo saber si se ha hecho contacto con lo real? Después de todo esta experiencia no puede realmente simbolizarse. La angustia es el único sentimiento que no puede ocultar el encuentro con lo real (Badiou, 2017). En el tratamiento el analista sitúa al individuo, por ejemplo, en esta posición, aunque no puede apresurar el sentimiento de la angustia ni retardarlo. El análisis trabaja con la angustia como el punto de acceso a lo real garantizado por la fórmula del conocimiento que es el matema. Observamos un tratamiento similar en Temor $y$ temblor (Kierkegaard, 2003) donde el elocuente pseudónimo titulado "Johannes de Silentio" busca situar (por no decir angustiar) al lector so pretexto de la histórica genésica del sacrificio de Isaac. Esta seña del psicoanálisis lacaniano es lo que le hace común a los diversos autores de la tradición antifilosófica.

La cuestión para la antifilosofía no es hacer una crítica intelectual acerca del correlato entre ser y pensar pues esto sería otro modo más de hacer filosofía. Desde este punto de vista: "la filosofía no solo se considera equivocada o refutada, sino que se muestra exhibida como una monstruosidad amenazante: enfermedad para Nietzsche, palabrería y verborrea sin sentido para Wittgenstein, estupidez y charlatanería para Lacan" (Boostels, 2008 , p. 163$)^{35}$. La antifilosofía muestra que el correlato entre ser y pensar el discurso filosófico es obsoleto para los propósitos de un acercamiento con lo real.
La antifilosofía destituye a la filosofía: mostrándole lo que su pretensión teórica ha perdido, y que no es, en definitiva, nada menos que lo real. Así, en el caso de Nietzsche, la vida es lo que llega como resto de todo protocolo de evaluación. Y del mismo modo en que la Caridad, para Pascal, se sustrae por entero del orden de las razones, la voz de la conciencia, para Rousseau, se sustrae a la predicación de la Luces, y la existencia, para Kierkegaard, a la síntesis hegeliana. En cuanto a Lacan, sabido es que el filósofo no quiere ni puede tener que conocer nada del goce y de la Cosa a la que está unido. (Badiou, 2013a, p. 34, [énfasis propio]).

Badiou habla de este resto, un remanente que persiste sin poder ser verbalizable ni simbolizable. El resto es inalcanzable y se sitúa más allá del lenguaje como el concepto mismo de vida en Nietzsche, aquello que no puede ser dicho por el Iohannes de Silentio del Temor y temblor kierkegaardiano y que por último alcanza su máxima expresión y constituye el centro interpretativo mismo del psicoanálisis bajo la categoría lacaniana de lo real.

Como fuera de toda simbolización este remanente es por definición indecible. El filósofo no puede mostrarlo. En cambio, el antifilósofo debe señalarlo a través de un gesto o un acto radical como la apuesta pascaliana, el salto de fe kierkegaardiano, la ruptura de la historia nietzscheana o la noción de acto en Lacan y Žižek (Boostels, 2008, p. 168). $\mathrm{Al}$ respecto hay que cuidarse de comparar el acto antifilosófico del concepto de "acontecimiento" en Badiou con el que se podrían comparar pues el acto antifilosófico no es una verdad impersonal sino un efecto (catártico o terapéutico) en el sujeto (Boostels, 2008, p. 168) ${ }^{36}$.

El antifílosofo habla a título personal y exige que "se exhiba constantemente como singularidad existencia" (Badiou, 2013a, p. 28). El antifilósofo expone públicamente su pensamiento privado.

¿Por qué? Porque, a diferencia del anonimato reglado de la ciencia, y en oposición a aquello que, en la filosofía, pretende hablar en nombre de lo Universal, el acto antifilosófico, sin precedentes y sin garantía, solo se tiene a sí mismo y a sus efectos como atestación de su valor. (Badiou, 2013a, pp. 28-29). 
Las obras antifilosóficas no son tratados ni sistemas (Boostels, 2008) sino que tienen un estilo personal como atestigua el Memorial de Pascal, las Confesiones de Rousseau o los diarios y obras edificantes de Kierkegaard.

Esta estrategia de hablar a título personal surge por el interés del antifilósofo de hablar con otros individuos qua individuos y no con otros filósofos (Badiou, 2013b, p. 46) ${ }^{37}$. Los antifilósofos no tiene la intención de discutir con filósofos y convencerlos de su postura pues en el mismo momento en el que comienzan a argumentar con filósofos los antifilósofos se convierten ipso facto en filósofos. Por esta razón lo que conviene a la antifilosofía, para propósitos de desarrollar su propio discurso, es ignorar a la filosofía. "Los antifilósofos piensan que no hay posibilidad alguna de conducir a los filósofos al acto" (Badiou, 2013b, p. 46) ${ }^{38}$. Badiou cita el ejemplo de Pascal quien más que dialogar con la filosofía especulativa de su tiempo "destina su identificación negativa de la filosofía al libertino. Con él es con quien hablamos" (Badiou, 2013b, p. 46) ${ }^{39}$. Pascal no se propone refutar al racionalismo.

El libertino no es Descartes, es otro personaje, otra configuración. Es él quien, tal vez, podría ser influenciado por Descartes, o caer bajo su influencia. El libertino es el que debe ser arrancado de la filosofía y devuelto al pensamiento verdadero, que es el cristianismo como lo piensa Pascal. (Badiou, 2013b, p. 46) $)^{40}$.

Lo mismo ocurre con Kierkegaard cuya obra fue interpretada por la academia como una crítica a Hegel pero que en realidad estaba destinada a la edificación personal y contrarrestar los efectos de la filosofía especulativa del hegelianismo danés, más nunca por medio de una crítica directa sino, a través, de comunicación indirecta (Stewart, 2003).

\section{ANTIFILOSOFÍA Y FILOSOFÍA EDIFICANTE}

Richard Rorty, en su célebre obra La filosofía y el espejo de la naturaleza, incluye una lista de pensadores como los señalados por Badiou, como Wittgenstein o Nietzsche, y algunos más cuyas intenciones y programa intelectual difieren de tal manera de la filosofía tradicional que a su parecer conviene realizar una distinción.
En su obra Richard Rorty hace una crítica contra la obsesión de la filosofía tradicional de querer traducir el conocimiento de la realidad en una epistemología. A este tipo de filosofía, que pone la epistemología en su centro, Rorty le llama filosofía sistemática. Esta filosofía

construida para la eternidad, ofrece argumentos diseñados para ser intrínsecamente indiscutibles a fin de hacer innecesaria la justificación y la defensa. Las obras de Aquino, Descartes, Hobbes, Newton, Russell y Husserl implican que la objetividad y la racionalidad son grandes virtudes epistemológicas que son apreciadas más allá de la superstición, el acuerdo o convención: y que existen realmente, incluso más allá de toda investigación. (Llanera, 2011, p. 109) ${ }^{41}$.

Rorty critica esta posición que no sale más allá de cierto estilo académico útil sólo para los especialistas. Los filósofos pueden dedicarse a entablar debates interminables y discutir sobre cualquier cosa

¿pero qué tan significativo es ese debate en términos de la práctica real, en términos de la vida misma? La visión franca y posiblemente correcta de Rorty de la mayoría de las controversias filosóficas centradas en lo epistemológico y en lo ontológico es que estos debates son demasiado a menudo (aunque no siempre) un callejón sin salida. (Saarinen, 2013, p. 151) ${ }^{42}$.

En oposición a la filosofía sistemática, cuyo intención es llegar al fondo de las cosas y convertirse en un reflejo de la naturaleza para alcanzar la verdad, Rorty identifica una serie de filósofos que deben ser reconocidos bajo otro nombre. Estos lanzan "provocaciones para que seamos más creativos, incluso experimentales, en nuestras interpretaciones de nuestras situaciones de vida" (Llanera, 2011, p. 109) ${ }^{43}$. A estos filósofos Rorty les otorga el nombre de edificantes quienes son, ante todo, escépticos hacia todo intento de proyecto de conmensuración universal (Rorty, $1983)^{44}$.

Rorty identifica que en la periferia de la historia de la filosofía moderna existe una serie de figuras que 
sin llegar a formar una 'tradición', se parecen entre sí por su desconfianza ante la idea de que la esencia del hombre es ser un conocedor de esencias. Pertenecen a esta categoría Goethe, Kierkegaard, Santayana, William James, Dewey, el segundo Wittgenstein, y el segundo Heidegger. Muchas veces se les acusa de relativismo o de cinismo. Suelen formular dudas sobre el progreso, y especialmente sobre la última pretensión de que tal o cual disciplina ha conseguido por fin aclarar hasta tal punto la naturaleza del conocimiento humano que la razón se extenderá ahora por todos las confines de la actividad humana. (Rorty, 1983, p. 332).

Estos autores resisten la idea de una reificación de la humanidad y que "aun cuando tengamos una creencia verdadera justificada sobre todo lo que deseemos saber, quizá lo único que tengamos sea conformidad con las normas del momento" (Rorty, 1983, p. 332). Esto es que lo que suele atribuirse como verdades universales y válidas para todos los tiempos no sea otra cosa más que el modo contemporáneo en que se describe la realidad tan contingente como la descripción de cualquier época pasada.

Esa Saarinen comenta que los filósofos edificantes no presentan argumentos como los sistemáticos sino más bien visiones. "Las visiones pueden ser esclarecedoras, inspiradoras, perturbadoras, agonizantes, reveladoras, personalmente relevantes y de hecho transforman la vida" (Saarinen, 2013, p. 147) ${ }^{45}$. Estas visiones resultan más útiles para la vida cotidiana que los argumentos pues "el hecho es que, aparte de unos pocos eruditos privilegiados, a casi nadie le importa. Las visiones inspiran, elevan, transforman vidas, desencadenan la renovación y crean una elevación mágica, porque las personas claman por significado" (Saarinen, 2013 , p. 147 ${ }^{46}$. Sin hacer ninguna referencia a la antifilosofía lacaniana Saarinen presenta una de sus mismas características en la filosofía edificante de Rorty: lo referente a la verdad.

Las visiones que presentan los pensadores edificantes

no reducen el discurso de la verdad; $y$, por lo tanto, carecen de interés para las prácticas dedicadas al discurso de la verdad: ese es el estado de cosas, argumenta Rorty, de la filosofía académica. Como resultado, muchas veces no logra un impacto visionario.

(Saarinen, 2013, p. 147) ${ }^{47}$.

El interés del pensamiento de Rorty radica en conducir la reflexión "en la vida como un arte pragmático dirigido al mejoramiento, al progreso moral y social, en un modo autocrítico de sus discursos y modos de acción" (Saarinen, 2013, p. $147)^{48}$. Rorty hace un llamado a una filosofía con causa que en lugar de contentarse con describir la vida lo que busca es más bien mejorarla (Saarinen, 2013) ${ }^{49}$.

Encontramos otra característica que hace similar la antifilosofía con la filosofía edificante de Rorty: lo referente a un tipo de comunicación personal y subjetiva. Mientras que los filósofos sistemáticos son constructivos y dan argumentos. "Los grandes filósofos edificantes son reactivos y presentan sátiras, parodias, aforismos. Saben que su obra perderá vigencia cuando se pase el periodo contra el que estaban reaccionando. Son intencionadamente periféricos" (Rorty, 1983, p. 334).

Los grandes filósofos sistemáticos, nos dice Rorty, "como los grandes científicos, construyen para la eternidad" (Rorty, 1983, p. 334) o como a Kierkegaard le gustaba decir sub aespecie aeternitatis y "quieren colocar su materia de estudio en el camino seguro de la ciencia" (Rorty, 1983, p. 334). En cambio "los grandes filósofos edificantes destruyen en beneficio de su propia generación" (Rorty, 1983, p. 334) y "quieren dejar un espacio abierto a la sensación de admiración que a veces causan los poetas" (Rorty, 1983, p. 334). Por estas actitudes los filósofos edificantes "se ríen de la imagen clásica del hombre, la imagen que contiene la filosofía sistemática, la búsqueda de la conmensuración universal en un vocabulario final" (Rorty, 1983, p. 333). Sin embargo, a pesar de la risa socarrona de los filósofos edificantes, estos no pretenden polemizar con los sistemáticos, pues

no matan a la filosofía; En cambio, dirigen su ruta no dictatorialmente. Poetizan nuestro entorno familiar a través de los términos desconocidos de sus invenciones, lo que nos permite proponer nuevos objetivos, nuevas palabras o nuevas disciplinas, abriendo posibilidades innovadoras con un horizonte ampliado en mente. La edificación nos saca de nuestro antiguo ser por el poder de lo 
extraño, para ayudarnos a convertirnos en nuevos seres. (Llanera, 2011, p. 110) ${ }^{50}$.

La filosofía edificante de Rorty y la antifilosofía de Lacan y Badiou poseen una serie de características tan afines que pensamos que ambas propuestas identifican con distintos nombres una misma tradición. Una lectora de Rorty incluso lo postula del siguiente modo: "(Rorty) rompe el discurso intelectual al ser flagrantemente antifilosófico" (Llanera, 2011, p. 108) ${ }^{51}$. Ambas posturas, antifilosofía y filosofía edificante, realizan una crítica: lo que Lacan identifica en filosofía como la unión entre el ser y el pensar en Rorty lo encontramos como la denuncia de la obsesión epistemológica de la filosofía por erigirse como un correlato especular de la naturaleza.

Sin embargo, ni antifilósofos ni pensadores edificantes buscan disputarse la verdad con la filosofía, pues esto les convertiría en filósofos, sino proponer algo más. La antifilosofía busca posicionar al individuo frente a la propia realidad de su existencia mientras que la lectura de Rorty de la filosofía edificante se constituye como una 'visión' que contribuye a la vida cotidiana del individuo. Para Rorty esta visión no reduce el discurso a la verdad sino que buscan presentar un horizonte de sentido. Análogamente, la antifilosofía según Badiou, coloca al individuo frente a la dimensión del acto donde se tiene la experiencia de un resto o remanente más allá de toda significación conceptual y de toda verdad en la dimensión de lo real. Tanto en antifilosofía como en filosofía edificante el pensador habla a título personal, pues su objeto es comunicarse siempre con un individuo, de forma íntima y subjetiva. Por estas razones creemos que los diagnósticos elaborados por la antifilosofía y la filosofía edificante respecto a ciertos pensadores se refieren a un mismo fenómeno.

\section{CONCLUSIONES}

En este trabajo presentamos la aportación del término antifilosofía realizada por Lacan misma que puede comprenderse como una crítica al discurso del amo; como el señalamiento de la insuficiencia del discurso filosófico centrado exclusivamente en la adecuación entre el ser y el pensar que, a su vez, mantiene la comprensión del saber cómo una asociación entre la verdad y el conocimiento. "La filosofía, que siempre se halagó de su coraje para pensar y un supuesto heroísmo hacía la verdad, se interpreta a la inversa como un malentendido común de lo real que nos habita" (Soler, 2006, p.124 ${ }^{52}$. Es precisamente aquel registro de lo real lacaniano lo que hace falta en el discurso filosófico. Sin embargo, Lacan no llegó a desarrollar este planteamiento sino que se contentó con identificar su propia práctica psicoanalítica como un discurso antifilosófico que no tiene el interés de oponerse a la filosofía sino situarse en la región de la palabra que señala y confronta aquello que por definición no se puede imaginar o simbolizar: lo real. "La realidad no es accesible, excepto a través de un punto fuera del discurso, más allá del logos" (Boni, 2011, p. 211) $)^{53}$.

Badiou retoma la antifilosofía lacaniana y reconoce que ciertas posiciones, como las de Pascal, Rousseau, Kierkegaard, Nietzsche o Wittgenstein, bien pueden identificarse como antifilosóficas. Es posible identificar en estos autores lo que Lacan señalaba: que hay algo que rebasa las verdades filosóficas y este resto o remanente es, por definición, inefable: lo real. Si la filosofía persiste como la identificación de la diada conocimiento-verdad, la antifilosofía integra tres términos, desde su punto de vista inseparables: conocimiento-verdad-real. La antifilosofía, pues, posee una conciencia sobre el registro que más allá de un non-sense como el célebre non sequitur de la lógica es más bien $a b$-sense: más que carente de sentido es del todo ajeno de sentido. El ab-sense es también absence (ausencia) absoluta de sentido.

Como una categoría de verdad fundada en el
vacío, el acto anti-filosófico de Badiou retira
la creencia religiosa a sus estados pre-subje-
tivos y pre-evidentes en los que la convicción
de una verdad no depende de la influencia
bíblica o cultural para la legitimidad, sino
que encuentra su autoridad en la pureza y
persistencia del pensamiento en relación
con lo infinito. (McCaffrey, 2017, p. 108)

La antifilosofía apunta a esta carencia absoluta de sentido sin la pretensión de reducirla al lenguaje pues sabe de antemano que esta empresa es imposible. En cambio, es un discurso abierta a la dimensión del acto donde los acontecimientos son más elocuentes que las palabras.

El trabajo de la antifilosofía, si queremos llamarlo así, implica prestar atención a estos momentos en que las identidades se 
rompen. Sin embargo, lograr esto requiere un pensamiento sin identidad, es decir, una forma de pensar que no está limitada por sus propias categorías, conceptos y posición institucional, sino que sigue las conexiones que la llevan hacia y desde otras formas de hacer, haciendo, y pensando. (Tanke, 2009, p. 226$)^{55}$.

Finalmente, realizamos una comparación entre la antifilosofía y el concepto de filosofía edificante de Rorty. El filósofo norteamericano distingue la filosofía sistemática de la filosofía edificante. La filosofía edificante no reduce la verdad al discurso, sino que apela más bien a la región de una experiencia subjetivísima y personal. Encontramos una coincidencia entre los autores que Rorty califica como edificantes y los pensadores catalogados como antifilósofos por Badiou. Más aún, aunque hay diferencias en los respectivos vocabularios el mayor encuentro entre estas posiciones es el objetivo planteado por la antifilosofía y la filosofía edificante. Ambas posturas se muestran como críticas a la filosofía pero no pretenden atacarla sino, nuevamente, mostrar su insuficiencia y dirigir el discurso a aquello que se resiste a ser simbolizado el remanente o resto que Lacan concibió como el registro de lo real. 


\section{Referencias}

Alemán, J. (2001). Introducción a la antifilosofía. Virtualia, $1(2), 1-9$.

Badiou, A. (1999). La fundación del universalismo. Barcelona: Anthropos Editorial.

Badiou, A. (2013a). La antifilosofia de Wittgenstein. Buenos Aires: Capital Intelectual.

Badiou, A. (2013b). Le Seminaire. Lacan. L'antiphilosophie 3. 1994-1995. Paris: Librairie Arthème Fayard.

Badiou, A. (2015). Le Seminaire. Nietzsche L'antiphilosophie 1. 1992-1993. Paris: Librairie Arthème Fayard.

Badiou, A. \& Cassin, B. (2017). There's No Such Thing as a Sexual Relationship. Nueva York: Columbia University Press.

Boni, L. (2014). Dare atto dell'impossibile: Badiou, Lacan e l'antifilosofia. Aut Aut, 363, 209-223.

Bosteels, B. (2008). Radical Antiphilosophy. Filozofski vestnik, 29(2), 155-187.

Clemens, J. (2013). Psychoanalysis is an Antiphilosophy. Edinburgh: Edinburgh University Press.

Djordjevic, C. (2009).What is Antiphilosophy? Metaphilosophy, $50(1-2), 16-35$.

Groys, B. (2016). Introducción a la antifilosofía. Buenos Aires: Eterna cadencia.

Johnston, A. (2010). This Philosophy Which Is Not One: JeanClaude Milner, Alain Badiou, and Lacanian Antiphilosophy. Journal of the Jan van Eyck Circle for Lacanian Ideology Critique, 3, 137-158.

Kierkegaard, S. (2003). Temor y temblor. Madrid: Alianza Editorial.

Lacan, J. (1992). El reverso del psicoanálisis, Seminario XVII. Buenos Aires, Barcelona, México: Paidós.

Lacan, J. (2012). Otros escritos. Buenos Aires: Paidós.

Lacan, J. (2008). El seminario de Jacques Lacan: libro 17: el reverso del psicoanálisis 1969-1970. Buenos Aires: Paidós.

Llanera, T. A. (2011). Shattering Tradition: Rorty on Edification and Hermeneutics. Kritike, 5(1), 108-116.
Masseau, D. (2000). Les ennemies des philosophes. L'antiphilosophie au temps des Lumieres. Paris: Albin Michel.

McCaffrey, E. (2017). Apostles of anti-philosophy: Badiou and Lyotard. French Cultural Studies, 28(1), 95-110.

Milner, J. C. (1995). La obra clara. Lacan, la ciencia, la filosofía. Buenos Aires: Ediciones Manantial.

Robertson, B. (2015). Lacanian Antiphilosophy and the Problem of Anxiety: An Uncanny Little Object. New York: Palgrave Macmillan.

Rorty, R. (1983). La filosofía y el espejo de la naturaleza. Madrid: Cátedra.

Saarinen, E. (2013). Kindness to Babies and Other Radical Ideas. En A. Groschner, C. Koopman, \& M. Sandbothe (Eds.), Richard Rorty, From Pragmatist Philosophy to Cultural Politics (pp. 145-154). Londres: Bloomsbury Academic.

Sharpe, M. (2015). Killing the father, Parmenides: On Lacan's anti-philosophy. Continental Philosophy Review, 1-24. doi: 10.1007/s11007-015-9330-8.

Soler, C. (2006). Lacan en antiphilosophe. Filozofski vestnik, 27(2), 121-144

Stewart, J. (2003). Kierkegaard's relation to Hegel reconsidered. Cambridge: Cambridge University Press.

Tanke, J. (2009). Reflections on the Philosophy and AntiPhilosophy of Art: Badiou and Rancière. Philosophy Today, 53(3), 217-230.

Tomsic, S. (2016). Psychoanalysis and antiphilosophy: the case of Jacques Lacan. En A. Cerda-Rueda (Ed.), Sex and Nothing: Bridges from Psychoanalysis to Philosophy (pp. 81-103). Lóndres: Karnac.

Tuggy, A. (1996). Léxico Griego-español del nuevo testamento. El Paso, Texas: Editorial Mundo Hispano.

Tutivén, C. (2002). Lacan y la filosofía. Iconos, Revista de Ciencias Sociales, FLACSO-Ecuador, 13, 48-55. doi: https:// doi.org/10.17141/iconos.13.2002.617 


\section{Notas al final}

1 "Mais, me direz-vous: que devient la philosophie dans tout cela ? Eh bien, la philosophie, c'est ce qui ne passe pas". 2 "It is both true and commonplace to observe that Jacques Lacan is by far the most philosophical of psychoanalytic thinkers".

3 "Antiphilosophical position is that because antiphilosophy is against philosophy, this means that the audience A that the antiphilosopher assumes she is contending with is philosophical".

4 "Dès 1972, «L 'étourdit » stigmatisait du terme d'imbécillité le kantisme attardé de tout »ce qui pense« de notre temps. Il désignait par là, les philosophes ou hommes de science induits par la « topologie inepte » de Kant qui dit que, je ne connais que des phénomène. Cette imbécillité est dénégation du réel".

$5 \quad$ "Antiphilosophy is not antiphilosophical in the sense of being 'non-philosophical'; on the contrary, the antiphilosopher is not out to evade or destroy philosophy, but to draw attention to forms of knowledge that philosophy cannot know, by affronting philosophy and subverting its claims".

6 "If anything, the 'anti' that I see at work in his antiphilosophical study of anxiety needs to be understood along the same lines that Orthodox Christians tend to read the 'anti' in Antichrist: What interests him is much less a direct, sustained, and fully visible assault on established philosophical doctrines than the subtle replacement of the latter with something new and imperceptibly — but undeniably_different".

$7 \quad$ "Badiou distinguishes antiphilosophy from philosophy on the basis of attitude, if not content or method. It is a type of thought that is constituted, if we are to believe Badiou, in a "hostility" to the systematic pretensions of philosophy proper".

8 "It deploys the logical and analytical tools of philosophy against itself in an effort to point to its tacit assumptions or systematic failures".

$9 \quad$ "Anti-philosophy thus characterizes those forms of thought that posit a founding act of intuition or unsayability, such as is to be found in Kierkegaard's critique of Hegel".

10 "This formulation leaves no doubt that the privileged target of antiphilosophy is not so much philosophy, but the university discourse, which places the quarrel in a different light" (Tomsic, 2016, p. 92). "Lacan flatly identifies "philosophical discourse in its true light' as a variation on the master's discourse" (Johnston, 2010, p. 140).

11 "Lacan's third claim concerning philosophy in The Other Side of Psychoanalysis is thus to associate philosophy with what he from this time now delineates as "the discourse of the masters". Philosophy would on this account be a way of seeking knowledge which, despite Plato's famous claim that the philosopher should ascend out of the political-historical cave (Rep. VII), would always remain subject to the needs of, if not the desire for, political rule (in the matheme of the discourse of the master, the S1 in the position of agency)".

12 "Le philosophe est donc celui qui tourne rond dans le discours du maître. Il y joue le rôle du fou. C'est-à-dire le tenant-lieu de la vérité, absolument inconscient de ce qu'il dit, et qui, par conséquent, n'est astreint qu'à tenir ce rôle'.

13 "Lacan's theory of discourses envisages in the university an intertwining of capitalism and scientific knowledge, and in its socio-political context the rise of bureaucratic power and the proliferation of experts".

14 "However, Lacan's genealogical account, typically bold and provocative, stands open to the claim that it commits the genetic fallacy, and also massively overreduces the plurality of historical philosophical practices and claims".

15 "Les analystes doivent lire de la philosophie, mais pour la mettre à l'épreuve sous la loi ultime du discours analytique, et donc la lire non pas pour rentrer dans la philosophie, mais pour savoir s'y soustraire".

16 La teoría de los cuatro discursos puede ubicarse en el seminario XVII (Lacan, 1992).

17 "The truth of psychoanalysis, Lacan by contrast always argues, is a founding dehiscence or split between subject and world. For psychoanalysis as an antiphilosophy, 'there is some relationship of being that cannot be known.' As Lacan puts it, evoking Parmenides' fragment: 'The discordance between knowledge and being is my subject'".

18 "In terms of Lacan's structuring categories, we have by now ventured that philosophic discourse in the classical form (which for Lacan is philosophy per se) forecloses from theoretical consideration the specific dimension of the symbolic, central to psychoanalysis. Language for the philosophers is the means of disclosure, hence of the possible adequatio or inadequation (in error, illusion, and falsity) with what is real. For Lacanian psychoanalysis, by contrast, words or signifiers are first of all the proffered tokens of a founding good faith between subjects, hence of the symbolic exchange in which deceit and betrayal become possible, and in which each of our desires is shaped".

19 Otra recepción del término antifilosofía es elaborado por Boris Groys quien en Introducción a la antifilosofía (2009) desarrolla el concepto a partir de una fuente distinta al psicoanálisis. Groys se inspira en los ready-mades de Marcel Duchamp y el movimiento anti-arte. Así como el anti-arte buscó irrumpir en el mundo establecido de las instituciones artísticas a través de la introducción de objetos cotidianos en el espacio de la galería, el antifilósofo incorpora la experiencia subjetiva a la práctica filosófica con el fin de poner en duda dicha práctica. Así, el orden temático y etimológico de antifilosofía es distinto para Groys de Lacan y Badiou, para quien la antifilosofía se nombra así por analogía al antiarte. Lo característico de este giro "que comienza con Marx y Kierkegaard, [es que] ya no opera por medio de la crítica sino por medio de órdenes" (Groys, 2016, p. 14). Para Groys la antifilosofía muestra la verdad solo una vez que se ha cumplido la orden. "Primero hay que transformar el mundo, y recién entonces el mundo se muestra en su verdad" (Groys, 2016, p. 14).

20 "The assumption that the question of being, or that of the world, is coextensive with the question of language; consequently, the reduction of truth to being nothing more than a linguistic or rhetorical effect, the outcome of historically and culturally specific language games or tropes which therefore must be judged and, better yet, mocked in light of a critical-linguistic, discursive, or genealogical analysis".

21 "It is crucial to appreciate here the difference between knowing/knowledge, as involving conscious acquaintance or 
familiarity (connaître/connaissance), versus knowing/knowledge (savoir) as entailing conceptual, intellectual comprehension”. 22 "The truths of the unconscious, situated in the register of the Real, defy connaissance but not (analytic) savoir. This savoir is what Lacan situates "between knowing" (connaître)-connaître inevitably brings with it the quotidian and philosophical temptations to render things recognizable through infusions of communicable meaning and significance, thus guaranteeing misrecognition (méconnaissance) of the meaningless contingencies (that is, Real truths) brought to light by analysis—“and being unaware".

23 "In Badiou's reconstruction of the Lacan of this period, the savoir that is neither conaissance nor directly vérité (although it sustains a relationship to the latter) is that which "touches ab-sense".

24 The real, in "L'Étourdit," is clearly definable on the basis of the absence of sense. As a result, in order for the truth/ knowledge/real triad to be thought through thoroughly it has to be shifted around in relation to the question of sense".

25 En El traje nuevo del Emperador de Hans-Christian Andersen observamos un absurdo. El emperador está desnudo y todo el reino pretende mirar su ropaje. Este non-sense es posible dentro de un campo de significación particular: monarquía, súbditos, reino, fábula, etc.

26 "The philosophical operation, for Lacan, consists in asserting that there is a meaning of truth. But why does philosophy claim there is a meaning of truth? Because its objective, the consolation it offers us under the name of "wisdom," is to be able to say that there is a truth of the real".

27 "The relation to the real that Lacan proposes as that of the discourse of the analyst will be a relation of a meaning of knowledge qua ab-sex sense, whereas the philosophical relation to the real is in the register of truth".

28 Cfr. "Led astray by that fatal symptom, the love of truth, philosophy fails to grasp the principle by which something of meaning is linked to knowledge in the function of the real, in what makes a formula out of "there's no such thing as a sexual relationship". Philosophy fails to grasp that absolutely unique sense that is, strictly speaking, neither sense nor non-sense but absex sense. This obviously means that philosophy fails to grasp the real, even in terms of the givenness of the real that consists in gaining access to a meaning of knowledge".

29 "Philosophy is trapped in the sense-truth pair, which assumes that the opposite of sense is non-sense, not ab-sense".

30 "Badiou subsequently emphasizes in this same essay that philosophy is both unwilling and unable to acknowledge and incorporate ab-sense".

31 "First, philosophy lacks any awareness of the register of ab-sense. It doesn't want to know anything about that register. Philosophy invariably substitutes something else for ab-sense or ab-sex sense. Thus, as Barbara Cassin has shown, Aristotle brings in the principle of noncontradiction in its place. [...] Second, philosophy knows nothing about the function of knowledge in the real. It absorbs it in the love of truth. [...] And third, there is a specular quality to philosophy because it arranges meaning and truth as mirror images under the guise of saying that there may possibly be a meaning of truth".

32 "The real is impossible to know (connaître) precisely because it is embedded in the truth-knowledge-real triad and can't be extracted from it in order to be paired with one of the other two terms. You even always have to say about the real that it "supplants knowing" (dépose le connaître). Lacan calls this supplanting of knowing the "demonstration" of the real, which is a

pretty strange word, albeit a very compelling one. The real cannot be known; it must be demonstrated".

33 Cfr. "Since there is neither knowledge (connaissance) nor truth of the real, and since, on the contrary, there is truth provided only that it is inextricably linked to the real in the guise of a knowledge (savoir) function, there has to be a pure encounter with this real. Let's call the point where the real as such is encountered an "act".

34 "Philosophy couldn't care less about this external decision, in which the act constitutes the silent incision of an unknown truth, since philosophy's distinctive temporal hallmark is that it has all the time in the world. This has always served to identify in its discourse the antidialectical connection it has with the eternity of Truth".

35 "Philosophy is considered to be not just refuted or mistaken but put on display as a threatening monstrosity: an illness for Nietzsche, chatter and nonsensical verbiage exhibiting itself as sense for Wittgenstein, stupidity and rascalness for Lacan".

36 "Unlike in Badiou's treatment of the "event," with which it is sometimes conflated, what matters in this "act" is not its impersonal truth so much as its - cathartic of therapeutic - effect on the subject".

37 "Un aspecto de la antifilosofía, a veces desatendido pero, en mi opinión, esencial en la estrategia antifilosófica, es que el antifilósofo habla siempre como si no tuviera interés en dirigirse a los filósofos". Un aspect de l'antiphilosophie, quelquefois négligé mais, à mon avis, tout à fait essentiel dans la stratégie antiphilosophique, est que l'antiphilosophe parle toujours comme s'il n'y avait aucun intérêt à s'adresser aux philosophes".

38 "Les antiphilosophes pensent qu'il n'y a aucune chance de rallier les philosophes à l'acte".

39 "C'est la raison pour laquelle chez Pascal, l'identification négative de la philosophie est destinée au libertin. C'est à lui qu'on parle".

$40 \quad$ "Et le libertin n'est pas Descartes, c'est un autre personnage, une autre configuration. C'est celui qui, peut-être, pourrait être influencé par Descartes, ou tomber sous son emprise. Le libertin est celui qu'il faut arracher à la philosophie et rendre à la pensée vraie, qui est le christianisme tel que le pense Pascal".

41 "Systematic philosophy puts epistemology at its center. Built for eternity, it offers arguments designed to be intrinsically unassailable so as to make justification and defense unnecessary. The works of Aquinas, Descartes, Hobbes, Newton, Russell and Husserl imply that objectivity and rationality -great epistemological virtues that are prized beyond superstition, agreement, or convention - truly exist, even if they may or may not be accessible through inquiry. But Rorty says that this kind of thinking is obsolete. Constructive philosophers have not effectively learned to contend with differences in contexts and cultural climates, for they refuse to fully embrace particulars, small-scale observations, and contingencies".

42 "Philosophers can create never-ending debates about anything and everything imaginable. But how significant is that debate in terms of actual practice, in terms of life itself? Rorty's blunt and quite possibly correct view of most epistemologically- 
and ontologically-focused philosophical controversies is that these debates are too often (though not always) a dead end".

43 "Rorty revaluates philosophy by believing that our problems can be conveniently distinguished into two ways: they are either attempts to get to the bottom of things, to mirror nature and achieve certainty; or, they are provocations for us to be more creative, even experimental, in our interpretations of our life-situations".

44 "Los filósofos que se mantienen dentro de la corriente principal son los filósofos a quienes llamaré 'sistemáticos', y a los periféricos los llamaré 'edificantes'. Estos filósofos periféricos, pragmáticos, son escépticos en primer lugar hacia la filosofía sistemática, hacia todo el proyecto de conmensuración universal”.

45 "The life and times of visions are different from those of arguments. Visions can be enlightening, inspiring, upsetting, agonizing, eye-opening, personally relevant, and indeed life-transforming".

46 The fact is that apart from a few privileged scholars, hardly anyone cares.' Visions inspire, elevate, transform lives, trigger renewal, and create magical uplift, because people cry out for meaning". En Esa Saarinen, "Kindness to Babies and Other Radical Ideas".

47 But there is a cost. The visions do not reduce to the discourse of 'truth; and thus fall short of being of interest to practices devoted to the discourse of 'truth: That is the state of affairs, Rorty argues in so many words, in academic philosophy. As a result, it oftentimes fails to achieve visionary impact".

48 "I submit that this is the categorical imperative of Rorty's thinking: focus on life as a pragmatic art directed to betterment, to moral and social progress, in a mode that is self-critical of its discourses and modes of action".

49 Cfr. "Rorty's call is for a philosophy with a cause. Instead of describing life, or debating such descriptions, he wants to nurture life, improve it, and make it emerge in more hopeful forms than currently is the case". Esa Saarinen, "Kindness to Babies and Other Radical Ideas".

50 "But Rorty thinks this accusation is a misunderstanding - for edifying philosophers do not kill philosophy; instead, they steer its route non dictatorially. They poeticize our familiar surroundings through the unfamiliar terms of their inventions allowing us to come up with new aims, new words, or new disciplines - opening innovative possibilities with an enlarged horizon in mind. Edification takes us out of our old selves by the power of strangeness, to aid us in becoming new beings".

$51 \quad$ "Richard Rorty (1931-2007) navigates in order to make his mark in the realm of philosophical thinking. He ruptures intellectual discourse by being flagrantly anti-philosophical: by avoiding the ironic act of asking essentialistic yet unanswerable questions, such as, What is being? What is human nature? What can we know for sure? Instead, Rorty restrategizes his approach toward our interpretation of ideas so as to downplay the Sisyphean search for primordiality; after all, he boldly exclaims that "philosophy" is just a pigeonhole into which different things are stuffed in different centuries, and even in different universities in the same country at the same time".

52 "Ainsi la philosophie, qui de toujours s'est flattée de son courage à penser et d'un supposé hérö̈sme de la vérité, se trouve-t-elle interprétée à rebours comme méconnaissance commune du réel qui nous habite".

53 "Il reale non è accessibile se non attraverso un punto fuori-discorso, extra-logos".

54 "As a category of truth founded in a void, Badiou's anti-philosophical act strips back religious belief to its pre-subjective and pre-evental states where conviction to a truth does not depend on scriptural or cultural leverage for legitimacy, but finds its authority in the purity and persistence of thought in relation to the infinite".

55 "The work, therefore, of anti-philosophy - if we are to call it that-involves attending to these moments where identities break down. Achieving this, however, requires a thought without identity, that is, a form of thinking that is not bound by its own categories, concepts, and institutional position, but instead follows the connections that carry it into and out of other ways of doing, making, and thinking". 